Richard Stang, Alexandra Becker, Fabian Franke, Christine Gläser, Anke Petschenka, Hans-Dieter Weckmann und Bert Zulauf

\title{
Herausforderung Lernwelt Hochschule
}

\author{
Perspektiven für eine zukünftige Gestaltung
}

\section{Einleitung}

Die Ergebnisse des Projektes Lernwelt Hochschule zeigen, dass die Hochschullandschaft in Deutschland derzeit in Bewegung ist, um auf die vielfältigen Herausforderungen der veränderten Lern- und Lehrstrukturen zu reagieren. Die Arbeiten des Centrums für Hochschulentwicklung (CHE) und des HIS-Instituts für Hochschulentwicklung (HIS-HE) weisen ebenfalls auf vielfältige Veränderungsprozesse in der Hochschullandschaft hin, die eine Reaktion auf die aktuellen gesellschaftlichen Herausforderungen sind. Dabei legen viele Hochschulen großen Wert auf eine stärkere Studierendenorientierung bei der Entwicklung ihrer Strategien zur Gestaltung der Lern- und Lehrumgebungen. Unter anderem die Aktivitäten des Stifterverbandes für die Deutsche Wissenschaft und des damit verbundenen Hochschulforums Digitalisierung (HFD) haben in den letzten Jahren vermehrt Impulse in diese Richtung gesetzt. So ist die Initiative Digitale Changemaker des Hochschulforums Digitalisierung ein wichtiger Schritt, die Studierenden einzubeziehen:

Lernende werden in den aktuellen hochschulpolitischen Debatten und wissenschaftlichen Diskursen rund um die Chancen und Herausforderungen des digitalen Wandels oft noch zu wenig mit eingebunden. Auf diese Weise kommt ihnen zumeist nur die Rolle von passiven Teilnehmenden zu, für innovative Ideen und neue Perspektiven gibt es bisweilen weder die Offenheit des Prozesses, noch eine grundlegende Bereitschaft, von den letztendlichen Nutzer(inne)n her zu denken und zu handeln. Einige hochschulische und außerhochschulische Strukturen, Projekte und Initiativen zeigen jedoch deutschland- und europaweitweit, wie innovativ und aktiv, aber auch kritisch-konstruktiv Studierende Digitalisierungsprozesse auf verschiedenen Ebenen mitgestalten wollen und können - wenn sie denn die Möglichkeiten hierzu erhalten. Aus diesem Grund hat das Hochschulforum Digitalisierung die Initiative \#DigitaleChangeMaker ins Leben gerufen und will Studierenden die Chance bieten, Hochschulbildung gemeinsam neu, kreativ und innovativ zu denken und Diskurse aktiv in einer spannenden Community von Expertinnen und Experten unterschiedlicher Hintergründe mitzugestalten. (HFD 2019) 
Im Rahmen der Recherchen des Projektes Lernwelt Hochschule wurde deutlich, dass sich immer mehr Hochschulen auf den Weg machen, den Shift from Teaching to Learning nicht nur im Rahmen von hochschuldidaktischen Veränderungen zu gestalten, sondern die Hochschule als gesamte Organisation zu verändern. Konkrete Umsetzungen und Handlungsempfehlungen werden im Band Zukunft Lernwelt Hochschule vorgestellt (Stang/Becker 2020). Im vorliegenden Band werden nun die Herausforderungen aufgezeigt, die sich auf Basis der Recherchen und Untersuchungen im Rahmen des Projektes Lernwelt Hochschule herauskristallisiert haben. Dabei sollen nicht nur die vier inhaltlichen Bereiche Hochschulorganisation, Hochschuldidaktik, digitale Strukturen und physische Lehr- und Lernräume in den Blick genommen werden. Ein Nadelöhr für die dynamische Entwicklung stellt auch die Hochschulpolitik dar, deren Einfluss auf die Entwicklung von „atmenden“ Hochschulen, in denen Lernende im Mittelpunkt strategischer Planungen stehen, eine zentrale Rolle spielt.

\section{Hochschulpolitik}

\section{Lernwelt Hochschule aufeinander abstimmen}

Hochschulpolitik ist in Deutschland aufgrund des Kultur- und Bildungsföderalismus vor allem Ländersache. Mit dem politischen Primat der Kulturhoheit als ein Teil der Eigenstaatlichkeit der Länder sollte nach dem Zweiten Weltkrieg auch die Hochschullandschaft gestaltet und entwickelt werden (Hildebrandt/ Wolf 2016, 35).

Mit dem Hochschulrahmengesetz (HRG) hat der Bund 1976 steuernd in die Hochschulangelegenheiten der Länder mit dem Ziel eingegriffen, länderübergreifende Regelungen zu treffen. Nach mehreren Novellierungen und der Föderalismusreform hat sich die Zusammenarbeit von Bund und Ländern wesentlich verändert, ohne die Unterschiede in den Hochschulgesetzgebungen ganz aufzuheben. Diese Unterschiede in den Landeshochschulgesetzen führen immer wieder dazu, dass Regelungen zum Beispiel bezogen auf das Qualitätsmanagement länderspezifisch ausfallen können (Weichert 2020a).

Die Herausforderung wird sein, die strategischen Ausrichtungen noch stärker für eine gemeinsame Lernwelt Hochschule in Deutschland zu bündeln. Erste Ansätze lassen sich hier heute schon finden. 


\section{Einheitliche Struktur von Entwicklungs- und Strukturplänen (ESPs) sowie Zielvereinbarungen schaffen}

Die unterschiedlichen gesetzlichen Regelungen sind unter anderem von Bedeutung, wenn es um die Aushandlungen und Entscheidungsmechanismen der Stakeholder geht. So unterscheiden sich zum Beispiel die Entwicklungs- und Strukturpläne (ESPs) in ihrem Aufbau und Inhalten, und Zielvereinbarungen sind eine individuelle Aushandlung zwischen dem jeweiligen Landesministerium und den Hochschulen (Weichert 2020b). Dies erschwert die Vergleichbarkeit der Rahmenbedingungen der Gestaltung der Lernwelt Hochschule und die Aufstellung von Benchmarks. Es wäre sinnvoll, einen bundesweiten Orientierungsrahmen für die Lernwelt Hochschule zu erstellen, um die Zielrichtungen besser vergleichen zu können. Es geht dabei weniger um die Vereinheitlichung, sondern um eine verstärkte Transparenz beziehungsweise Offenlegung.

\section{Zielvereinbarungen als Stellschrauben nutzen}

Als große Stellschraube erweisen sich die einzelnen Zielvereinbarungen, welche zwischen Hochschulen und Länderministerien geschlossen werden. Diese Zielvereinbarungen sind ein Instrument des New Public Managements. Durch die Verschiebung der normativen Grundvorstellung von dem juristisch geprägten Verständnis der Erfüllung von Vorgaben hin zur Effektivität und Effizienz wird die Zielvereinbarung zum Messinstrument für die Wirtschaftlichkeit (Ziegele 2006, 78-79). Dabei ist allerdings entscheidend, welche Prioritäten gesetzt werden und welche Dimensionen bei der Gestaltung von Zielvereinbarungen einbezogen werden. Dies erfordert eine differenzierte Analyse der Rahmenbedingungen, das heißt der Situation der Stakeholder - extern wie intern - und des Umfelds, in dem sich die Hochschule bewegt. Allerdings besteht hier auch die Gefahr, dass gegebene Leistungen in keinem direkten Bezug zu der erbringenden Gegenleistung stehen, dass zu wenig Entscheidungsspielräume bei den Hochschulen verbleiben oder die finanzielle Relevanz der Zielvereinbarung unklar formuliert ist (Ziegele 2006, 87-91). Die Herausforderung wird sein, eine Balance herzustellen, die allen Hochschulen auch eine individuelle Schwerpunktsetzung ermöglicht. 


\section{Unterschiedliche Steuerungsmodelle berücksichtigen}

Entscheidend ist darüber hinaus, welches Steuerungsmodell in der Hochschule präferiert wird. Die Vielfalt an Steuerungsmodellen birgt einige Herausforderungen für die Lernwelt Hochschule in sich. Im Bereich der Educational Governance lässt sich die Lernwelt Hochschule als Mehrebenensystem beschreiben. Sobald zwischen diesen Ebenen gleichzeitige Interdependenzen vorliegen, wird Handlungskoordination erforderlich (Niedlich 2020, 30), die das Vorankommen der Hochschulen innerhalb des Innovationsprozesses verlangsamen und mit erheblichen Aufwänden verbunden sein kann.

Eine der bedeutsamsten Veränderungen war die Einführung der leistungsorientierten Mittelvergabe (LOM). Durch die Einführung wettbewerbsorientierter Mechanismen, anhand derer die finanziellen Mittel verteilt werden, sollen Anreizstrukturen geschaffen werden. Die Output-Orientierung sorgt dafür, dass Ressourcen in Abhängigkeit eines vereinbarten oder eines bereits erreichten Zieles verausgabt werden. Diese Steuerung erfolgt über Formelmodelle (Mittelverteilungsmodelle), durch Zielvereinbarungen und durch Einzelvergaben finanzieller Mittel aus Finanz- und Stellenpools (Hüther et al. 2011, 21-22). Damit unterliegen einzelne Hochschulen unterschiedlichsten finanziellen Rahmenbedingungen. Hinzu kommt, dass vornehmlich Drittmittel für die Forschung eingeworben werden, die oftmals die Lehre mitfinanzieren (müssen), da diese eingeworbenen Drittmittel für die Zuteilung von Mitteln seitens des Ministeriums relevant sind. Die zentrale Herausforderung wird vor dem Hintergrund einer studierendenorientierten Hochschulentwicklung sein, die Maßstäbe für Studierendenorientierung zu operationalisieren und Bewertungskriterien zu entwickeln.

\section{Selbstorganisation optimieren}

Die Art der Steuerung und Selbstorganisation der Hochschulen sind zentrale Elemente der Hochschulautonomie. Doch die Umsetzung der Etablierung von Bottom-up-Prozessen erfordert Handlungskoordination, die im Rahmen der Veränderungsprozesse in der deutschen Hochschullandschaft noch nicht stark entwickelt ist. Da jede Hochschule individuell auf der Suche nach Lösungen ist, sowohl inhaltlich als auch für die Prozesse und Strukturen, liegen hier bislang keine fundierten Daten vor. So fehlt bislang ein deutschlandweiter strukturierter und systematisierter Überblick zur Thematik Studierendenorientierung der Hochschulen. Vor allem das Zusammenspiel und der Austausch der verschiede- 
nen Akteurinnen und Akteure der Hochschule scheinen noch entwicklungsfähig (Becker/Stang 2020b; Aschinger 2020). Hier fehlen Studien und der Austausch der Akteurinnen und Akteure auf den verschiedenen Ebenen, denn damit könnten Erfahrungen und Erkenntnisse für alle Beteiligten transferiert werden und sich für die einzelnen Hochschulen als hilfreich erweisen.

\section{Finanzierungssituation verbessern}

Finanzierung ist eine weitere Dimension der Hochschulautonomie. Doch wird diese Autonomie nach wie vor in unterschiedlicher Weise durch Länderministerien beschränkt (Dilger 2015, 1), wobei sich auch hier in einigen Ländern Veränderungen in Richtung einer Stärkung der Autonomie feststellen lassen (Dohmen/Krempkow 2015, 64). Doch um eine stärkere Autonomie umsetzen zu können, bedarf es zusätzlicher Finanzmittel. Am Beispiel des Qualitätspakts Lehre lässt sich zeigen, dass im Zusammenspiel von Bund und Ländern zwar Investitionen getätigt werden, diese aber nur als Projektfinanzierung kurzfristig Aktivitäten fördert. Die langfristige Sicherung von Innovationen in der Lernwelt Hochschule im Rahmen einer gesicherten Zuwendung steht hier längst aus. Hier bedarf es neuer Finanzierungskonzepte. Die Entwicklung veränderter Finanzierungsmodelle auf der einen Seite sowie die Ausweitung des Finanzierungsvolumens - vor allem auch im Hinblick auf die internationale Konkurrenzsituation auf der anderen Seite, stellen große Herausforderungen für die Hochschulpolitik dar.

\section{Bologna-Prozess weiterentwickeln}

Zwanzig Jahre nach dem Start des Bologna-Prozesses kann festgehalten werden, dass sich dieser Prozess noch immer im Werden befindet. Der Europäische Hochschulraum ist auf der einen Seite näher zusammengerückt, auf der anderen Seite sind sehr unterschiedliche Entwicklungen festzustellen (Blättler/Imhof 2019). Während die Bachelor-/Master-Studienstruktur weitgehend umgesetzt ist, werden die Konkretisierungen doch insgesamt länderspezifisch unterschiedlich gehandhabt. Stellen die einen die Studierenden- und Kompetenzorientierung in den Fokus des alltäglichen Handelns, orientieren sich andere an bürokratischen Leistungsmessungsstrukturen. Gleichzeitig wurde - zumindest in Deutschland - nicht selten versucht, das Pensum eines Diplomstudiengangs in den Bachelorstudiengang zu zwängen. Auf der politischen Ebene scheint der Bologna-Prozess kein zentrales Thema mehr zu sein. Doch zeigen sich auf der 
Hochschulebene noch immer Verwerfungen, die durch politische Rahmensetzungen bearbeitet werden sollten. Unter der Perspektive der Gestaltung der Lernwelt Hochschule ergibt sich für die Hochschulpolitik die Herausforderung, den Bologna-Prozess unter einer veränderten Perspektive - der Studierendenorientierung - neu zu vermessen und zu gestalten.

\section{Reformprozesse fördern}

Insgesamt lässt sich für den Bereich Hochschulpolitik - der obersten Ebene des Mehrebenensystems Hochschule - festhalten, dass sich die Reformprozesse in Bezug auf die Lernwelt Hochschule derzeit im Modus der Suchbewegung befinden. Vieles ist auf Bundesebene in Angriff genommen worden, wie zum Beispiel mit dem Hochschulpakt 2020 oder dem Qualitätspakt Lehre, auch die Länder legen immer wieder Programme auf, die zur Verbesserung der Lernwelt Hochschule beitragen sollen. Doch fehlt es hierbei nach wie vor an einer Gesamtstrategie, die die Entwicklung auch langfristig sichert. Hier sind andere Länder Deutschland voraus (Weichert/Stang 2020). Für Hochschulpolitik besteht die Herausforderung, diese Prozesse nicht durch noch mehr bürokratische Verwaltungsabläufe, sondern durch Innovationskorridore zu unterstützen. Dazu bedarf es auch einer transparenteren Struktur von Daten.

\section{Transparente Datenstruktur erzeugen}

Auch wenn viele Daten bezogen auf die Hochschulentwicklung in Deutschland erhoben werden, wurde im Rahmen des Projektes Lernwelt Hochschule deutlich, dass es an vielen Stellen an vergleichbaren Daten fehlt. So lagen bislang keine umfassenden Studien zur Lernwelt Hochschule unter der Perspektive Studierendenorientierung vor. Eine bundesweite Übersicht über die Struktur physischer Lehr- und Lernräume an deutschen Hochschulen ist auch nicht zu finden. Auch fehlt ein strukturierter Überblick über die Weiterentwicklung der Lehre. Die Frage nach den methodischen Settings und der didaktischen Gestaltung ist aber für die Weiterentwicklung der Lernwelt Hochschule von zentraler Bedeutung. Eine öffentlich zugängliche Übersicht über Zielvereinbarungen und Entwicklungsund Strukturpläne wäre zumindest für den staatlich verantworteten Bereich der Hochschulen wünschenswert.

Auch wenn die Bildungsberichtserstattung viele Daten liefert (Autorengruppe Bildungsbericht 2018, 151-172), stellt es unter der Perspektive der Gestaltung der Lernwelt Hochschule eine Herausforderung dar, differenzierte Informationen 
über eingesetzte Lehrmethoden und die Gestaltung von digitalen und physischen Lehr- und Lernräumen zu erhalten.

\section{Entwicklung dynamisieren}

Betrachtet man die Entwicklung im internationalen Kontext (Weichert/Stang 2020), wird deutlich, dass Deutschland in Europa eher zu den „latecomern“ in Bezug auf eine innovative Entwicklung der Lernwelt Hochschule gehört. Zwar gibt es vielfältige Initiativen, doch fehlt es der Entwicklung an Dynamik. Die Komplexität hochschulpolitischer Entscheidungsprozesse, bürokratische Hindernisse und nicht zuletzt eine traditionelle Hochschulkultur wirken bremsend. Es verwundert von daher gerade nicht, dass derzeit an privaten Hochschulen, die sich am Markt behaupten müssen, ein anderes Innovationsklima herrscht als dies an staatlichen Hochschulen der Fall ist. Die Herausforderung für die Hochschulpolitik ist in diesem Zusammenhang, die Entwicklung zu dynamisieren und nicht durch Intensivierung bürokratischer Strukturen $\mathrm{zu}$ entschleunigen. Dazu bedarf es aber auch einer Neuausrichtung der Hochschulorganisation.

\section{Hochschulorganisation}

\section{Gesamtstrategie in den Blick nehmen}

Auffallend ist, dass es bezogen auf die Gestaltung der Lernwelt Hochschule bislang an Hochschulen nur wenige Ansätze gibt, eine Gesamtstrategie und damit ein Gesamtkonzept für eine studierendenorientierte Hochschule $\mathrm{zu}$ entwickeln. Dies zeigt sich, wenn man die Bereiche Hochschuldidaktik und digitale Strukturen oder auch die Entwicklung der physischen Lehr- und Lernräume in den Blick nimmt (Aschinger 2020; Becker/Stang 2020b; Gläser/Kobsch 2020). Nicht selten werden hier in Hochschulen jeweils einzelne Konzepte entwickelt, diese jedoch (noch) nicht strukturiert aufeinander abgestimmt beziehungsweise miteinander verwoben. Das daraus resultierende Problem ist zum einen, dass das Gesamtergebnis für die Studierenden vermutlich hinter dem Optimum zurückbleibt, und zum anderen, dass viele Parallelstrukturen und -prozesse entstehen, die Ressourcen kosten, die an einer anderen Stelle innerhalb der Organisation sinnvoll genutzt werden könnten. Ein drittes Problem sind hier auch die Reibungsverluste bezogen auf Information und Kommunikation zwischen den Beteiligten 
(Becker/Stang 2020b). So werden Ergebnisse zur Weiterentwicklung der Lehre nicht hochschulweit kommuniziert. Die vorhandenen Kommunikationsformen sind nur bedingt dazu geeignet, die Erkenntnisse nachhaltig in der gesamten Hochschule zu verbreiten und zu etablieren (Becker/Stang 2020b). Dieser Umstand kann zum einen der gewünschten Dezentralisierung des New Public Managements, zum anderen aber auch dem traditionellen Verständnis von $\$ 5$ Abs. III GG - der Freiheit von Forschung und Lehre - geschuldet sein. Kulturell gewachsene Selbstverständnisse der Akteurinnen und Akteure sind hier sicher ein zentraler Aspekt im Zusammenhang mit der Schwierigkeit, eine ganzheitliche Gesamtstrategie zu entwickeln und zu verankern, was eine der zentralen Herausforderungen für Hochschulen darstellt.

\section{Ungleichgewicht von Forschung und Lehre beseitigen}

Eine Schieflage bei der strategischen Ausrichtung von Hochschulen - und hier besonders Universitäten - stellt das Ungleichgewicht der Bewertung von Forschung und Lehre dar. Während sich Forschungsexzellenz für die Forschenden beziehungsweise Lehrenden in Form von hochschulinterner Reputation niederschlägt und auch mit unterschiedlichen Gratifikationen Wertschätzung erhält, fristet Lehrexzellenz - trotz vielfältiger Lehrpreise - noch immer ein Schattendasein. Problem in diesem Zusammenhang ist die Beantwortung der Frage, nach welchen Kriterien exzellente Lehre bewertet werden kann. Während es im Forschungsbereich eindeutige Kriterien gibt, wie Drittmitteleinwerbung, referierte Fachaufsätze und dergleichen mehr, fehlt für den Bereich der Lehre ein solches Kriterienraster. Die Freiheit von Forschung und Lehre führt deshalb gerade im Bereich der Lehre, in dem das Gegenüber die Studierenden mit ihren vielfältigen und divergierenden Anforderungen sind, zu der problematischen Situation, dass oft mit der Freiheit der Lehre argumentiert wird, obgleich es nachweislich an pädagogischer Qualität fehlt und nicht sehr viel Energie investiert wird. Auch hier hat sich in den letzten Jahren vieles zum Positiven entwickelt, doch für eine strategische Ausrichtung der Hochschule in Richtung Studierendenorientierung bedarf es weiterhin verstärkter Bemühungen.

\section{Strategische Hochschulentwicklung gestalten}

Auch wenn unter der Perspektive des New Public Managements Dezentralisierung in den Fokus rückt, bedarf es umso mehr einer „Kopplung von organisationalen Bestandteilen durch Transparenz, Messbarkeit und zurechenbare Verant- 
wortlichkeit (,Accountability')“ (Koch 2009, 122-123, H. i. O.). Für die Hochschulen stellt die strategische Planung zwischen tradierter Organisationskultur und den neuen Erfordernissen einen Spagat dar. Zwar wird in Hochschulen oft vom Generationenwechsel gesprochen, doch scheint es äußerst schwierig, tradierte Strukturen aufzubrechen und die Selbstverständnisse der verschiedenen Stakeholder zu wandeln. So ist die Öffnung der Hochschule auch im Kontext Public Private Partnership (PPP) ein Beispiel für veränderte Strukturen, das unterschiedlich bewertet werden kann. Im Ausland ist dies selbstverständlich (Weichert/Stang 2020). So können auf der einen Seite in der Lehre Projekte mit realer Aufgabenstellung in Partnerschaft mit Wirtschaftsunternehmen studiengangübergreifend und interdisziplinär realisiert werden. Dadurch erhalten Studierende praktische Einblicke in die zukünftige Arbeitswelt, gleichzeitig haben die Unternehmen die Möglichkeit, Studierende zu rekrutieren. Auf der anderen Seite stellt sich dabei die Frage, nach der Abhängigkeit in der Lehre von den Unternehmen. Für die Hochschulorganisation erfordert es hier eine strategische Entscheidung und - wenn die Entscheidung pro Public Private Partnership fällt klarer Beschreibungen von Strukturen und Prozessen bei der Zusammenarbeit. Dazu zählen Punkte wie Finanzierung, Anpassung der Beschreibungen in den Modulhandbüchern, Vereinbarkeit mit den beteiligten Studiengängen, Verstetigung und Dauer von Partnerschaften. Die Herausforderung, die sich daraus ergibt, ist, die Gestaltung der Strategie im Netzwerk aktueller und potentieller Stakeholder auszutarrieren.

\section{Leitbilder sowie Entwicklungs- und Strukturpläne präzisieren}

Die Gestaltung der strategischen Ausrichtung wird in Leitbildern sowie Entwicklungs- und Strukturplänen dokumentiert (Becker/Stang 2020; Weichert 2020b). Die Perspektive der Output-Orientierung, die sowohl auf dem Konzept der kompetenzorientierten Lehre, dem Bologna-Prozess wie auch dem New Public Management basiert, ist ein wichtiges Element für die strategische Ausrichtung der Lernwelt Hochschule. Bislang werden die meisten „Outcomes“ in Kennzahlen fixiert, die für die Beurteilung im Rahmen der Zielvereinbarungen und Entwicklungs- und Strukturplänen erforderlich sind. Die quantitative Betrachtung ist dabei nur eine Seite der Medaille. Es stellt sich die Frage, wie qualitative Kriterien einbezogen werden können, die nicht nur Aussagen über das, was gemacht worden ist, treffen lässt, sondern auch darüber, wie es gemacht wird. Hochschulen haben zwar damit begonnen, Leitbilder Lehre zu entwickeln, unter einer studierendenorientierten Perspektive wäre es allerdings genauso wichtig ein Leitbild Lernen der Weiterentwicklung der Lernwelt Hochschule zugrunde zu 
legen. Insgesamt scheint in der (Neu-)Justierung der Dokumente Leitbild und Entwicklungs- und Strukturplan eine wichtige Herausforderung zu liegen, zumal in die Entwicklung der strategischen Dokumente meist nur eine eingeschränkte Anzahl von Hochschulakteurinnen und -akteuren einbezogen sind (Becker/ Stang 2020b).

\section{Beteiligung der Hochschulakteurinnen und -akteure sicherstellen}

Die Ergebnisse des Forschungsprojektes Lernwelt Hochschule zeigen, dass die Forderung nach stärkerer Partizipation der Organisationsmitglieder der Hochschule noch nicht auf breiter Ebene umgesetzt ist. Ist die aktive Einbindung auf der Ebene der Mitarbeitenden in Form von Arbeitsgruppen, Gremien oder Workshops noch relativ einfach zu gestalten, so ist die Einbindung von Studierenden in den strategischen Entscheidungsbildungsprozess der Hochschule schwieriger. Dieser Herausforderung wird über Fragebögen, Evaluationen sowie die Einbindung von Vertreterinnen und Vertretern in Gremien zu begegnen versucht (Becker/Stang 2020b). Doch scheint es neuer Konzepte und Ideen zu bedürfen, um die Studierenden besser in den Strategieprozess einzubinden. Gleichzeitig zeigt sich auch, wie schwierig es ist, Studierende für die Diskussion über die Zukunft der Lernwelt Hochschule zu aktivieren. Hier scheint die Entwicklung neuer Anreizsysteme erforderlich, wie es zum Teil durch Ideenwettbewerbe oder ähnlichem schon versucht wird (Aschinger 2020). Weitere Ansatzpunkte werden in dem Verhältnis der Studierenden zu ihrer Hochschule, dem Wissen über Partizipationsmöglichkeiten und über die Hochschule als Organisation, aber auch der wahrgenommene Zeit- und Leistungsdruck der Studierenden gesehen (Gläser/Kobsch 2020). Dies verweist wieder auf die Bedeutung der Kommunikation von strategischen Zielen und der Information über diese im Hinblick auf alle Hochschulakteurinnen und -akteure, insbesondere der Studierenden. Die Entwicklung eines Partizipationsmodells ist dabei eine zentrale Herausforderung.

\section{Zusammenarbeit der Hochschulakteurinnen und -akteure intensivieren}

Doch nicht nur die Information zu und Kommunikation über die strategischen Ziele ist von Relevanz, sondern auch die Beantwortung der Frage, wie die ver- 
schiedenen Hochschulakteurinnen und -akteure die Umsetzung dieser Ziele gemeinsam angehen. Derzeit scheinen Hochschulen noch wie eine Inselgruppe zu sein, bei der es bei „Ebbe“ wohl begehbare Wege zwischen den einzelnen Inseln gibt, bei „Flut“ aber alle auf ihren Inseln verharren. Die Gestaltung dieser Wege ist kein triviales Unterfangen, da die verschiedenen Verwaltungsabteilungen, Fakultäten, Institute und nicht zuletzt auch die Lehrenden sehr unterschiedliche Vorstellungen und Kulturen bezogen auf die Lernwelt Hochschule entwickelt haben. Diese miteinander in einen produktiven Austausch zu bringen, dürfte eine der größten Herausforderungen bei der Gestaltung der Lernwelt Hochschule sein. Dazu bedarf es der Entwicklung eines Kooperationsmodells bezogen auf die verschiedenen Hochschulakteurinnen und -akteure sowie externe Stakeholder.

\section{Zugänglichkeit der Hochschule ermöglichen}

Ein weiterer Aspekt, der die Hochschulorganisation und die Studierenden betrifft, ist die Zugänglichkeit der Hochschule. Dies umfasst sowohl die Gebäude an sich, die physischen Lehr- und Lernräume, aber auch Services wie Ausleihe in der Bibliothek und Nutzung von Lernplattformen. Ein Ergebnis des Forschungsprojektes Lernwelt Hochschule ist, dass sich die Kodierungen für diese Zugänglichkeiten auf diverse Schlüssel, wie Hochschulausweis, Matrikelnummer etc. verteilt. Dies ist für Studierende nicht immer intuitiv nachvollziehbar. Die $\mathrm{Zu}$ gangskonzepte sind oft der Verwaltungslogik der Hochschule geschuldet und zum Teil historisch gewachsen (Becker/Stang 2020b).

Unter einer studierendenorientierten Perspektive betrachtet, stellt die $\mathrm{Zu}$ gänglichkeit der Lehr- und Lernräume und des Gebäudes einen wichtigen Faktor für eine flexible Studienorganisation dar. Auch die Zugänge zu Ausleihen, ob nun in der Bibliothek oder aber bezogen auf Technik und Material, bedürfen einer einheitlichen Struktur. Auch die Zugänge zu digitalen Services erfordern oft unterschiedliche Anmeldeprozeduren (Becker/Stang 2020b). Die Konsolidierung von Anmeldeprozeduren ist ein weniger komplexes Unterfangen, welches effektiv und effizient den Studierenden in ihrem Alltag hilft. Ebenso lassen sich die zahlreichen Anmeldeoptionen zu digitalen Angeboten der Hochschulen auf eine einzige Kennung zusammenführen. Auch dies dürfte eine Entlastung für die Studierenden darstellen, die kostengünstig und unkompliziert zu realisieren ist. 


\section{Hochschule als multidimensionale Organisation koordinieren}

Hochschule ist eine multidimensionale Organisation, die eine komplexe Handlungskoordination erfordert. So sind die verschiedenen Logiken und Kulturen von Verwaltung, Forschung, Lehre, IT-Abteilungen, Infrastrukturabteilungen usw. miteinander zu koordinieren und zu moderieren. Gleichzeitig gibt es in diesen verschiedenen Sphären wiederum Strukturen, die die Komplexität noch vergrößern. So sind zum Beispiel im Forschungsbereich Forschende zum einen verbeamtete Professorinnen und Professoren, zum anderen unbefristet beschäftigte aber auch befristet beschäftigte Mitarbeitende, die sich wiederum in Forschungs-, Verwaltungs- und technisches Personal unterteilen lassen. Wenn dann noch wissenschaftliche Mitarbeitende bei den Professorinnen und Professoren promovieren, dann addiert sich eine weitere Abhängigkeitsstruktur, die zu bearbeiten ist. Ähnliches lässt sich für die Lehre deklinieren, die von verbeamteten Professorinnen und Professoren, fest und befristet angestellten Mitarbeitenden und externen Lehrbeauftragten realisiert wird. Kommen dann noch die Studierenden ins Spiel, multipliziert sich die Komplexität. Die verschiedenen Perspektiven und Interessenslagen machen es so schwierig, Hochschulorganisation ganzheitlich zu gestalten. Dies allerdings zu tun, ist für die Zukunft von Hochschulen eine zentrale Herausforderung.

\section{Ganzheitliche und integrierte Lernwelt entwickeln}

Es erstaunt vor diesem Hintergrund nicht, dass es bei dieser multidimensionalen Struktur äußerst schwierig ist, eine ganzheitliche und integrierte Lernwelt für die Studierenden zu gestalten. Vielmehr nehmen die Studierenden die Lernwelt Hochschule nicht selten als segmentiert und additiv wahr (Gläser/Kobsch 2020). Um diese Problemlagen in den Griff zu bekommen, bedarf es eines Blicks auf die Lernwelt Hochschule unter der Perspektive der Studierenden. Die Strukturen und Prozesse sollten zunächst aus der Perspektive der Studierenden geplant und dann im Abgleich mit den institutionellen Strukturbedingungen und Prozessabläufen weiterentwickelt werden. Die Lernwelt Hochschule sollte eben nicht nur aus der Perspektive der Produzentinnen und Produzenten, sondern auch aus der Perspektive der Nutzenden - also der Studierenden - betrachtet werden, wobei auch zu berücksichtigen gilt, dass es die Studierenden nicht gibt, sondern die Bedarfe und Bedürfnisse sehr unterschiedlich sind. Allerdings zeigen Entwicklungen im Ausland, dass ein solcher Zugang gleichwohl dazu führt, dass alle Beteiligten von Innovationen profitieren (Weichert/Stang 2020). 
An den internationalen Erfahrungen gilt es anzuknüpfen, um auch in Deutschland die Perspektiven auf die Lernwelt Hochschule zu verändern.

\section{„Atmende“ Hochschule denken}

Die Gestaltung der Lernwelt Hochschule erfordert von Seiten der Hochschulorganisation einen multiperspektivischen Zugang. Dabei spielen die Dimensionen Strategie, Struktur und Kultur eine zentrale Rolle. In diese Dimensionen eingewoben sind die internen Stakeholder (Mitarbeitende auf allen Hierarchieebenen), die passageren Stakeholder (Studierende) und die externen Stakeholder (Beratungsgremien, Alumni, Politik, Unternehmen usw.). Diese bilden den Organismus Hochschule. Dieser sollte so organisiert werden, dass er sich ,atmend“ an sich immer schneller verändernde Rahmenbedingungen anpassen kann. Die „atmende“ Hochschule wird - wenn sie die Lernwelt Hochschule für die Zukunft entwickeln will - Hierarchien reduzieren, Strategien mit allen Stakeholdern gemeinsam entwickeln, die Kommunikation zwischen den Stakeholdern strukturieren, eine Kultur der Integration etablieren und letztendlich die Studierenden in den Fokus aller Überlegungen stellen.

\section{Hochschuldidaktik}

\section{Shift from Teaching to Learning in den Fokus rücken}

Die klassischen Lehrformate wie Vorlesung und der traditionelle seminaristische Unterricht spielt in den Hochschulen nach wie vor die größte Rolle. Allerdings setzen sich immer stärker didaktische Ansätze durch, die offener konzipiert sind und das Lernen in den Fokus rücken. Dazu gehören zum Beispiel projektorientierte Veranstaltungen, in denen Projekte unter verschiedenen Perspektiven bearbeitet werden. Forschungsorientierte Konzepte führen Studierende anhand konkreter Problemstellungen in Forschungsmethoden ein. Konzepte auf der Basis des problembasierten Lernens (PBL) setzen auf kollaboratives Arbeiten. Zentrale Elemente des problembasierten Lernens sind:

- die Studierendenzentrierung,

- das Lernen in Gruppen,

- die veränderte Rolle der Lehrenden hin zur Lernbegleitung,

- Fragestellungen, die Anreiz für das Lernen schaffen, 
- die Entwicklung von Problemlösekompetenz,

- die Aneignung von Wissen durch selbstgesteuertes Lernen.

Ein solcher Zugang erfordert veränderte Lehr-Lernszenarien, in denen weniger die Vermittlung von Wissen im Fokus steht, sondern mehr die gemeinsame Erarbeitung. Dabei spielt die Arbeit in Projekten eine zentrale Rolle, in denen keine Lehrenden-Lernenden-Hierarchie mehr gelebt wird, sondern ein kollegialer Teamgedanke im Fokus steht. Diese veränderte Perspektive in den Blick zu nehmen, dürfte für viele Hochschulen derzeit eine große Herausforderung darstellen. Dazu kommt, dass sich vor diesem Hintergrund auch die Rolle der Lehrenden verändert.

\section{Verändertes Rollenverständnis der Lehrenden etablieren}

Wenn die Lernenden in den Fokus von Lernangeboten an den Hochschulen rücken, dann haben die Lehrenden die Aufgabe, den Prozess des Lernens zu organisieren, zu initiieren und zu begleiten. Die Lehrperson schafft den didaktischen Rahmen, in dem die Lernenden ihr Lernen gestalten können, da sie letztendlich selbst entscheiden, was sie lernen. Dies erfordert ein verändertes Verständnis von Lehren, das die Ermöglichung von Lernen ins Zentrum rückt.

Allerdings ist eine solche Orientierung didaktischer Settings für die Lehrenden voraussetzungsreich. Während die klassische Lehre stark strukturiert ist und sich input-orientiert gut steuern lässt, erfordern offene Lernstrukturen eine umfassendere Planung, da flexibel auf unterschiedliche Anforderungen reagiert werden muss. Lehrende werden zu Mitlernenden, die zwar Expertise einbringen, aber die Struktur der individuellen Lernprozesse nur mitgestalten, nicht vorgeben können. Lernprozesse werden so zu Kommunikationsprozessen, die durch Vielfalt der Zugänge, Lernwege und Ergebniskonstruktionen geprägt sind.

Informationen werden aus dieser veränderten didaktischen Perspektive nicht mehr von den Lehrenden vermittelt und von den Lernenden in einer Konsumhaltung zur Kenntnis genommen und eher passiv generiert, sondern Wissen und Kompetenzen werden aktiv generiert. Da die wenigsten Lehrenden an Hochschulen eine pädagogische Ausbildung haben, dürfte sich dieser Perspektivenwechsel nur sehr langsam etablieren. Für die Hochschulen stellt sich die Herausforderung, wie sie Prozesse der Fortbildung für die Lehrenden forciert, besonders, wenn diese kein Interesse an einer Veränderung haben. 


\section{Wissensgenerierung und Kompetenzentwicklung neu gestalten}

In diesen kommunikativen Strukturen findet Lernen durch gemeinsame Generierung von Wissen statt. Klassische Lehrangebote wie Vorlesung haben zwar weiterhin ihre Berechtigung im Portfolio der Lehrangebote, doch ist Wissensgenerierung durch den Konsum von Information nur begrenzt möglich. Vielmehr geht es darum, Wissen und Kompetenzen in Kommunikation mit anderen zu generieren beziehungsweise $z u$ entwickeln. Die Entwicklung vom Konsumieren zum Generieren ist eine Entwicklung, die auf allen gesellschaftlichen und ökonomischen Feldern zu finden ist. Die digitalen, mobilen Medien mit ihren vielfältigen Möglichkeiten zur Produktion von visuellen Materialien werden diese Entwicklung beschleunigen, da sie auch für (Forschungs-)Projekte vielfältige Optionen eröffnen. Die Lernwelt Hochschule sollte auf diese Veränderungen reagieren und Konzepte in diesem Bereich fördern. Die strategische Gestaltung von Methodenvielfalt müsste dabei ein zentrales Anliegen sein. Dies bedeutet allerdings auch, dass sich die Lehrenden hier offen zeigen und ihre Rolle als Pädagoginnen und Pädagogen wahrnehmen.

\section{Didaktikzentren als Schlüsseleinrichtungen anerkennen}

Der Positionierung von Didaktikzentren wird dabei in Zukunft eine besondere Rolle zukommen. Ihnen obliegt die Aufgabe, Lernende und Lehrende unter anderem methodisch aus- und weiterzubilden. Doch stellt sich die Frage, welche neuen Aufgaben hinzukommen, die sich zum Beispiel mit der Gestaltung von Lehr- und Lernmedien, Supervision der Einzelstudierenden und Kleingruppen, Lern- und Lehrcoaching, Beratungskompetenzen der Lehrenden und der Vermittlung von Didaktik befassen? Didaktikzentren sind vor allem im Zuge des Qualitätspakts Lehre ${ }^{1}$ eingerichtet beziehungsweise ausgebaut worden. In einigen Hochschulen haben sie noch Projektcharakter, was in Zusammenhang mit der deren strategischen Position in der Lernwelt Hochschule problematisch ist. Im Prinzip müssten sie eine abgesicherte Querschnittsposition in der Hochschulorganisation einnehmen. Dies vor allem auch deshalb, weil sie auf konzeptioneller Ebene fachübergreifende Verteiler für Innovation in der Lehre, Unterstützer der Curriculum-Entwicklung und des Qualitätsmanagements Lehre und für die Forschung zum Beispiel mit Methodenwissen zum forschenden Lernen und des Publizierens mit Schreibberatungen sein könnten. Auf personeller Ebe-

1 https://www.bmbf.de/de/qualitaetspakt-lehre-524.html. 
ne könnten sie die didaktische Qualität der Lehrenden sichern, neue Methoden in die Lehre hineintragen sowie ein Forum zur Reflexion und zum fachübergreifenden Austausch bieten. Auf der Organisationsebene könnten die Didaktikzentren nicht nur das Verständnis von Lehrqualität fördern, sondern auch einen Beitrag zur Personalentwicklung leisten.

Die größte Herausforderung aus Organisationssicht besteht darin, diese Einrichtung zu verstetigen, da sie an einigen Hochschulen aus zeitlich begrenzten staatlichen Fördermitteln, wie zum Beispiel im Rahmen des Qualitätspakts, finanziert werden. Eine weitere Herausforderung besteht darin, diese Einrichtung so in der Organisation zu verankern, dass sie zum einen genug Schnittstellen zu den relevanten anderen Abteilungen (IT-Abteilung, Infrastruktur-Abteilung etc.) hat und anderseits fachübergreifend agieren kann und damit in direktem Kontakt zu Studiengangsbeauftragten beziehungsweise Dekaninnen und Dekanen, aber auch zu den Hochschulleitungen hat.

\section{Leitbild Lehre als Qualitäts- und Kulturmerkmal positionieren}

Noch immer herrscht das Primat der Forschung an vielen deutschen Hochschulen. Der Blick auf die Lehre war auch vor dem Hintergrund von Aktivitäten wie der Exzellenzinitiative des Bundes teilweise in den Hintergrund geraten. Unter der Perspektive einer Gleichwertigkeit der Aufgaben (Forschung, Lehre, Third mission) bedarf es verstärkter Anstrengungen, den Bereich der Lehre innerhalb der Lernwelt Hochschule wieder stärker in den Fokus zu rücken, auch wenn Forschung finanziell lukrativer erscheint und deren Reputation auch durch veränderte Verteilmechanismen von Ressourcen zugenommen hat. Um die Lehre an Hochschulen zu stärken, sind in den letzten Jahren verstärkt staatliche aber auch private Förderprogramme aufgelegt worden, wie zum Beispiel der Qualitätspakt Lehre des Bundesministerium für Bildung und Forschung (BMBF), das Programm „Bologna-Zukunft der Lehre“2 der Volkswagen- und der MercatorStiftung, der Wettbewerb „Exzellente Lehre“3 von Kultusministerkonferenz und dem Stifterverband für die Deutsche Wissenschaft. Die vielfältigen Förderprogramme können allerdings nur einen externen Anreiz darstellen, die Schaffung von entsprechenden Strukturen liegt in der Hand der Hochschule.

Das Projekt Lernwelt Hochschule zeigt, dass bei nahezu drei Viertel der Befragten strategische Veränderungen im Bereich der Hochschuldidaktik stattgefunden haben oder geplant sind (Becker/Stang 2020b) und ein Viertel gab an,

2 https://www.volkswagenstiftung.de/sites/default/files/downloads/MB_86a_d.pdf.

$3 \mathrm{http} / / /$ www.exzellente-lehre.de/. 
die strategische Ausrichtung in einem Leitbild zu veröffentlichen (Becker/Stang 2020b). Ein genauerer Blick zeigt jedoch, dass nur wenige Hochschulen ein explizites Leitbild für die Lehre entwickelt haben. Die Entwicklung eines solchen Leitbilds wäre aber sinnvoll, um die Hochschule dahingehend zu positionieren, dass sie als (Gesamt-)Organisation ein Bewusstsein für gute Lehrqualität entwickelt und damit die Attraktivität der Hochschule für Studienbewerberinnen und -bewerber erhöht. Hochschulintern könnte es zu einer Steigerung einer dauerhaften (Lehr-)Qualitätskultur beitragen. Hochschulen sollten - unter Einbezug aller Akteurinnen und Akteure (Didaktikzentren, Qualitätsmanagement, Lehrende und Studierende) - ein Leitbild Lehre entwickeln, um gute Lehre hochschulintern zu verankern und die Möglichkeit für die Außendarstellung zu nutzen.

\section{Digitale Strukturen}

\section{Verbesserung der Schnittstellen gewährleisten}

Der digitale Wandel hat als gesamtgesellschaftliche Herausforderung starke Auswirkungen auf viele Lebensbereiche, insbesondere auch auf den Bildungsbereich. So hat die Digitalisierung in den letzten Jahren bereits weite Teile der Hochschullandschaft erfasst. In den Kernbereichen Lehre und Forschung wie auch in der Administration existieren heute eine Reihe digitaler Plattformen, die durch hochschulübergreifende Communities unterstützt werden. Solche Plattformen wie beispielsweise Learning-Management-Systeme und Campus-Management-Systeme wachsen strukturell stärker zusammen und werden auch stärker im Zusammenhang wahrgenommen. Zukünftige digitale Strukturen profitieren demnach von guten Schnittstellen zwischen den Plattformen und Systemen. Hochschulen im 21. Jahrhundert benötigen eine Durchdringung digitaler Strukturen auf allen Ebenen und den Abbau von strukturellen Barrieren damit der Übergang von Lehre zur Forschung oder das Zusammenspiel zwischen Lehre, Forschung und Administration effizient, aber auch effektiv abgebildet werden kann.

Als zentrale Herausforderung für die Verbesserung der Hochschulverwaltung und für die Umsetzung von Service- und Dienstleitungskonzepten dürfte sich in den nächsten Jahren insbesondere die Integration digitaler Technologien in der Lehre als eine zentrale Aufgabe ergeben. 


\section{Veränderungsprozesse mit Blick auf Digitalisierung gestalten}

Die Digitalisierung bietet Potentiale insbesondere bei der Profilbildung und Sichtbarkeit, als auch bei der Verbesserung der Hochschullehre (HFD 2016). Die Ausgestaltung ist zugleich Chance und Herausforderung. Chancen nutzbar zu machen, setzt voraus, Veränderungsprozesse aktiv zu gestalten. Dazu zählen das neue Rollenverständnis von Lehrenden und Lernenden, die Entstehung neuer Professionen in der Entwicklung der Lehre sowie die Bereitstellung einer modernen Infrastruktur und neuer Finanzierungsmodelle. Herausforderungen sind beispielsweise die Anrechnung digitaler Lehre auf das Lehrdeputat sowie eine verstärkte Kompetenzorientierung.

Zunächst sind Strategie- und Veränderungsprozesse im Lehr- und Lernkontext anzustoßen, die sinnvolle Nutzung von Technologien steht hierbei im Fokus. Ziel ist die Ausschöpfung von Potentialen digitaler Bildung. Dazu bedarf es auf hochschulstrategischer Ebene entsprechender Entscheidungen. So wurden in den letzten Jahren Strategiepapiere veröffentlicht, die deutlich machen, dass zukünftig mehr digitale Lehr- und Lernformate erprobt werden sollen. Um die Hochschulöffentlichkeit zu adressieren, können zum Beispiel E-Learning-Netzwerktage organisiert werden, um eine bessere Sichtbarkeit bereits realisierter Projekte und Service- und Dienstleistungen zu ermöglichen.

\section{Profilbildung und Kooperationen entwickeln}

Profilbildungen und Kooperationen innerhalb der Hochschule oder mit anderen Hochschulen sind weitere zentrale Faktoren, um digitale Strukturen effizient aufzubauen. Mittels eines Anreizsystems der kapazitativen Anrechnung von digital angereicherter Lehre können Lehrende ermutigt werden, neue Lehrformate zu konzipieren und zu erproben. Anreize können auch die Ausschreibung von Lehrpreisen und anderer prestigeträchtigen Angebote sein, in denen der Einsatz von digitalen Lehr-Lern-Szenarien im Fokus stehen. Insbesondere bei Berufungen können Hochschulen die Fokussierung auf Digitalisierung - digitalen Angeboten in der Lehre - als Stellschraube nutzbar machen. Die Investition in Unterstützungsstrukturen - beispielsweise den zentralen Einrichtugen - spielt laut dem Bericht des Hochschulforums Digitalisierung weiterhin eine zentrale Bedeutung (HFD 2016).

In den vorliegenden Ergebnissen des Forschungsprojekts Lernwelt Hochschule kommen ähnlich gelagerte Themen zur Sprache. So spielen die IT-Infrastruktur, das Campus-Management-Konzept, der Bereich E-Learning und E-Lehre 
eine zentrale Rolle. Die Herausforderung wird sein, die verschiedenen Strukturen miteinander zu vernetzen.

\section{IT-Infrastruktur als zentrale Stellschraube in den Blick nehmen}

Informationstechnologie ist für Hochschulen ein unverzichtbarer Bestandteil nahezu aller Arbeitsabläufe; so sind Forschung und Lehre wie auch Verwaltungsprozesse heute in hohem Maße von Informationstechnologie abhängig. Die Anforderungen an Informationstechnologie, bezogen auf Anzahl, Umfang, Verfügbarkeit, Komplexität und Sicherheit der angebotenen Services und Anwendungen sind stetig gestiegen. Durch den Einsatz von Informationstechnologie als „wichtiges Werkzeug bzw. Dienstleister der Prozessunterstützung“ (Gadatsch 2014, 31) haben sich insbesondere die Anzahl und der Umfang von ITServices an Hochschulen deutlich vergrößert.

Innovationen, die in der Informationstechnologie in immer kürzeren Abständen an Relevanz gewinnen, und der zunehmende Einsatz und die Nutzung von Informationstechnologie an Hochschulen fordern das IT-Management heraus. Jedes größere Forschungsprojekt ist gegenwärtig auch immer ein IT-Projekt (HHU 2015, 2-6). Lehrende und Lernende nutzen moderne Informationstechnologie zur Kommunikation und zum Austausch von Informationen in Text-, Bild-, Ton- und immer häufiger Videodaten (HFD 2016, 102-103, 111). Verwaltungsprozesse, wie etwa die Organisation des gesamten akademischen Lebenszyklus der Studierenden (Student Lifecycle), werden durch Campus-Management-Systeme als Online-Workflows abgebildet, beispielsweise für Einschreibung, Veranstaltungsbelegung, Prüfungsanmeldung oder die Anzeige erbrachter Studienleistungen.

\section{Digitale Perspektive in Entwicklungs- und Strukturplänen präzisieren}

Die strategischen Ziele von Hochschulen werden beispielsweise in NordrheinWestfalen aus Zielvereinbarungen mit der Landesregierung als Hochschulentwicklungspläne für jeweils fünf Jahre formuliert. Übergreifend zu den Entwicklungsplänen der Hochschulen gibt so zum Beispiel der Landeshochschulentwicklungsplan NRW einen allgemeinen Planungsrahmen für Hochschulen des Landes vor. Dieser Entwicklungsplan sieht Handlungsfelder mit starkem IT-Be- 
zug in den Bereichen Digitalisierung der Lehre (Landesregierung NRW 2016a; Landesregierung NRW 2016b, 29-31), international wettbewerbsfähige Forschung (Landesregierung NRW 2016b, 32-36), die Zusammenarbeit bei IT-Plattformen (Landesregierung NRW 2016b, 52) und in den Bereichen Governance und Prozesse vor (Landesregierung NRW 2016b, 53). Die Hochschulentwicklungspläne greifen diese Ziele auf und konkretisieren und erweitern sie $\mathrm{zu}$ individuellen Zielen der jeweiligen Hochschule. Die Notwendigkeit der digitalen Transformation von Hochschulen ist erkannt und wird vielfach durch konzeptionelle ITStrategien verankert.

Insgesamt ist eine der Herausforderungen in diesem Bereich, dass das Thema Digitalisierung in den relevanten Papieren wie Leitbildern, Hochschulentwicklungsplänen etc. nicht nur thematisiert, sondern die Umsetzung einer digitalen Strategie mit konkreten Maßnahmen unterfüttert wird. Die Bundesländer sind hier auf dem Weg, die Hochschulen werden veränderte Konzepte entwickeln.

\section{IT-Strukturen zwischen Zentralisierung und Dezentralisierung austarieren}

Die Aufteilung von Verantwortlichkeiten zwischen dezentraler und zentraler ITVersorgung ist ebenso wie die Zusammenstellung und der Ausbau des IT-Portfolios regelmäßig Bestandteil der IT-Strategie an Hochschulen. Die Gewichtung von IT-Portfolio-Themen und IT-Verantwortlichkeiten fällt jedoch sehr unterschiedlich aus und ist zum Teil geprägt von individuellen aktuellen Themen einzelner Einrichtungen. Die unterschiedlichen IT-Verantwortlichkeiten werden zum einen auf der einen Seite an stark dezentralen IT-Organisations-Strukturen oder auf der anderen Seite an sehr zentral orientierten IT-Organisations-Strukturen deutlich.

Es ist nachvollziehbar, dass im Rahmen der jeweiligen Hochschulkultur sehr unterschiedlich auf die Herausforderungen reagiert wird. Doch egal, welcher Weg beschritten wird, bedarf es einer Organisationsstruktur, die Aktivitäten auch im Hinblick auf die Studierenden bündelt. Konzepte wie die Etablierung eines Chief Information Officer (CIO), der die Abstimmung zwischen den verschiedenen IT-Verantwortlichen und die strategische IT-Portfolio-Gestaltung organisiert, könnte hier ein entsprechendes Konzept sein. Doch stellt sich für jede Hochschule die Herausforderung, ein an die eigene Hochschulkultur angepasstes Konzept zu entwickeln. 


\section{Campus-Management-Systeme als zentrale Instrumente integrieren}

Campus-Management-Systeme existieren in unterschiedlichem Umfang an allen Hochschulen und je nach Ausprägung werden die unterschiedlichen Hauptprozesse des Student Lifecycle in einem Reifegrad digital abgebildet. Die CampusManagement-Systeme unterstützen die sogenannte digitale Organisation des Studiums durch Prozesse, die als digitale Workflows, wie beispielsweise die Anmeldung von Prüfungen oder Abschlussarbeiten, die Anmeldungen zu Veranstaltungen oder die Anzeige erbrachter Studienleistungen, abgebildet werden. In Abbildung 1 werden die Aspekte einer Prozesslandkarte Campus Management aufgezeigt.

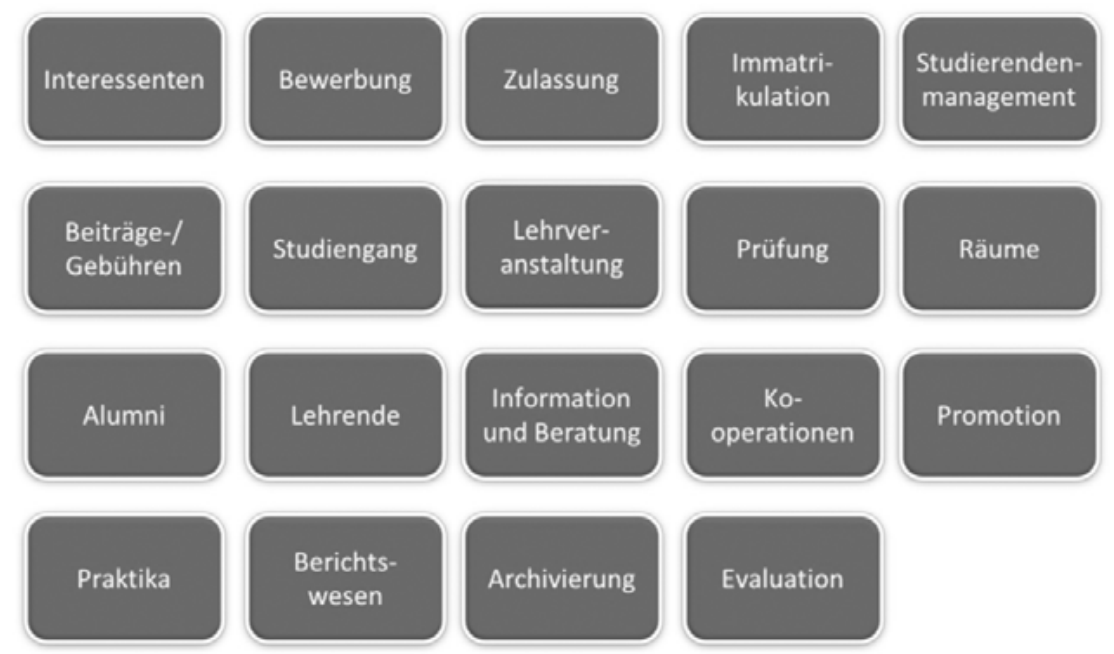

Abb. 1: Prozesslandkarte Campus Management (ZKI 2016, 4)

In vielen Fällen sind solche Campus-Management-Systeme bereits länger im Einsatz und stellen, mit der zunehmenden Digitalisierung und den zunehmenden Anforderungen an die Organisation und die Informationstechnologie, über die Jahre gewachsene Systeme dar. Die effektive Weiterentwicklung oder die Einführung eines neuen Campus-Management-Systems stellt viele Hochschulen vor zeitaufwändige und umfangreiche Projekte mit erheblichen Anforderungen an das Projektmanagement, an die Kommunikation mit allen relevanten Beteiligten und an die effiziente Umsetzung der neuen (digitalen) Prozesse. 


\section{E-Learning in den Fokus rücken}

Lehr- und Lernangebote mit digitalen Medien zu unterstützen, wird von vielen im Projekt Lernwelt Hochschule befragten Hochschulen zwischenzeitlich als selbstverständliche Maßnahme betrachtet (Becker/Stang 2020b). Sowohl in den Service- und Dienstleistungsstrukturen, als auch in den Fakultäten werden neue Lehr- und Lernformate erprobt. Allerdings kann hier keine flächendeckende Erprobung und Nutzung von modernen Lehr- und Lernformaten festgestellt werden. Sicher liegt dies auch daran, dass ein gesamtgesellschaftlicher Wandel zwar von allen Zielgruppen als Herausforderung anerkannt wird, allerdings die finanziellen und personellen Kapazitäten nicht hinreichend sind. Auch die noch kritische Abwägung hinsichtlich des Mehrwerts von digital angereicherter Lehre ist nicht selten ein Grund dafür, dass es nur langsam zu einem verstärkten Einsatz kommt. Wenn beispielsweise die Nutzung eines Lern-ManagementSystems bereits als ausreichender Ansatz digital unterstützter Lehre betrachtet wird, wird spätestens deutlich, dass auch die didaktische Perspektive eine zentrale Rolle spielt. So ist die alleinige Bereitstellung von Lehr- und Lernmaterialien nicht Sinn und Zweck von digitaler Lehre (HFD 2016). Vielmehr geht es neben grundsätzlichen Fragen, wie digitale Technologien Probleme der Hochschulen lösen und sie sich Chancen zunutze machen, vor allem um die ernsthafte Auseinandersetzung bei der Gestaltung innovativer Lehr- und Lernszenarien unter Einbezug digitaler Medien.

Allerdings zeichnet sich an vielen Hochschulen ab, dass projektbasierte Förderungen (BMBF, DFG, Stifterverband usw.) nicht unbedingt dazu führen, nachhaltige Strukturen $\mathrm{zu}$ etablieren. Positive Projektergebnisse werden oftmals nicht flächendeckend bekanntgemacht und in nachhaltige Strukturen und Angebote überführt. Hier sind zugleich politische als auch hochschulpolitische Strukturen zu optimieren.

Aus der Perspektive der Studierenden stellt die teilweise nicht optimal entwickelte E-Learning-Infrastruktur ein Problem dar, da nicht auf alle technischen Möglichkeiten zugegriffen werden kann. Seit Jahren ist bekannt, dass Studierende gerne E-Learning-Angebote nutzen. Für Hochschulen stellt sich die Herausforderung, in Zukunft eine an die Bedarfe der Studierenden angepasste $E$ Learning-Infrastruktur zu entwickeln. 


\section{Physische Lehr- und Lernräume}

\section{Raumgestaltung als strategischen Fokus erkennen}

Hochschulen stehen in steigendem Maße vor der Aufgabe, Lernmöglichkeiten und Arbeitsplätze in der Hochschule für ihre Studierenden zu erweitern und neue Lern- und Lehrräume zu schaffen. Das Zusammenkommen von steigenden Studierendenzahlen und dem Sanierungsstau auf der einen Seite (Becker/Stang 2020b) und den Anforderungen aus den hochschuldidaktischen Veränderungen auf der anderen Seite, stellt die Hochschulen finanziell und strukturell vor große Herausforderungen. Gleichzeitig fehlt es nicht selten an einer ausgewiesenen Strategie der Raumentwicklung. Diese zu gestalten ist eine der zentralen Herausforderungen, besonders auch vor dem Hintergrund der Digitalisierung (Günter et al. 2019).

\section{Lehr- und Lernumgebungen an Lernbedürfnissen und Lernprozessen orientieren}

Lehr- und Lernumgebungen an den Lernbedürfnissen und Lernprozessen der Studierenden zu orientieren, ist im Hinblick auf die physischen Lehr- und Lernräu$m e$ besonders wichtig. Nur wenn sie der Ausgangspunkt der konkreten Gestaltung von physischen Lehr- und Lernräumen sind, entstehen schlüssige Konzepte, die für Lernende Sinn machen und genutzt werden. Nach wie vor müssen die Studierenden - trotz der zunehmenden Digitalisierung - mit ihren analogen Bedürfnissen ernst genommen werden (Stang 2017). Für das gemeinsame Arbeiten an Projekten oder das Lernen in Gruppen wird von vielen Studierenden nach wie vor der physische Raum präferiert.

Die Aufenthaltsqualitäten wirken sich direkt auf den Studienalltag aus. Über die Infrastrukturen hinaus entstehen atmosphärische Qualitäten, die die sozialen und emotionalen Bedürfnisse der Lernenden befriedigen (Gläser/ Kobsch 2020). Auch die im Rahmen der Studie ausgewerteten internationalen Praxisbeispiele (Weichert/Stang 2020) belegen, dass physisch-räumliche Studienbedingungen die Kooperation, Kreativität und Identifikation im Studium befördern können. Die Herausforderung für die Hochschulen besteht darin, vielfältige Lehr- und Lernraumangebote zur Verfügung zu stellen, was nicht mit einem standardisierten Vorgehen zu realisieren ist. 


\section{Lehr- und Lernräume differenzieren, zonieren und flexibilisieren}

Internationale, aber auch nationale Praxisbeispiele zeigen deutlich, dass das Repertoire an Konzeptelementen für physische Lehr- und Lernräume bereits sehr umfangreich ist. Es gibt vielfältige Umsetzungen für differenzierte Arbeitsplätze, zum Beispiel für Einzel- und Gruppenarbeitsplätze oder Gruppenräume in verschiedenen Größen mit Präsentationsmöglichkeiten (Aschinger 2020). Zonierungen spielen bei der Strukturierung von physischen Räumen eine wichtige Rolle, um unterschiedliche Nutzerbedarfe zu berücksichtigen (DINI 2013). Auch innovative und explorative Konzeptelemente wie Makerspaces oder Learning Labs werden erprobt. Die Einrichtung von Lernräumen mit flexibler Möblierung spielt zunehmend eine größere Rolle. Ein hoher und komfortabler Standard an IT-Ausstattung, technischer Infrastruktur und Netzanbindung wird dabei vorausgesetzt. Die räumlichen Besonderheiten der einzelnen Hochschulen machen individuelle Konzepte notwendig, um die Anforderungen der Studierenden optimal zu erfüllen.

Als Herausforderung in diesem Zusammenhang zeigt sich die Gestaltung der Anpassung an sich verändernde Bedürfnisse. Dies erfordert neue Raumund Möblierungskonzepte, die sich an den Erkenntnissen der Lernforschung und an didaktischen Erfordernissen orientieren.

\section{Zugänge und Übersicht verbessern}

Der Bedarf an mehr studentischen Arbeitsplätzen mit vielfältiger Ausstattung, an besseren Informationen, wo freie Arbeitsplätze zur Verfügung stehen, und an Raumbuchungssystemen ist auf der strategischen Ebene der Hochschulen angekommen und wird bereits in vielfältigen operativen Maßnahmen adressiert (Aschinger 2020). Die Umfrageergebnisse im Rahmen des Projektes Lernwelt Hochschule zeigen sehr deutlich das Handlungsniveau in diesem Bereich, 31 Prozent der Hochschulen haben bereits Veränderungen vorgenommen, weitere 39 Prozent planen diese (Becker/Stang 2020b). Lernraumlandkarten, die eine Übersicht über die vorhandenen Lernräume und ihre Ausstattung bieten, und Informationssysteme, die die aktuelle Belegung anzeigen, können den Studierenden in den Hochschulen helfen, für sie in ihrer jeweiligen individuellen Lernsituation geeignete freie Lernräume und Arbeitsplätze zu finden (Aschinger 2020). Raumbuchungssysteme, die die Reservierung von Einzel- oder Gruppen- 
räumen ermöglichen, steigern Transparenz und Komfort für die Studierenden und können die Belegung von Räumen erheblich steigern.

Die Herausforderung, die sich hier ergibt, bezieht sich auf die Frage, wie die Zugänge und die Übersicht bezogen auf Lehr- und Lernräume an Hochschulen verbessert werden können, um die Nutzungsoptionen zu vergrößern und die Nutzung zu optimieren.

\section{Hybride Raumstrukturen entwickeln}

Das Zusammenspiel von digitalen und physischen Lehr- und Lernräumen ist bislang nur wenig entwickelt. Für die Zukunft wird es eine große Herausforderung sein, hier sind hybride Raumkonzepte $\mathrm{zu}$ entwickeln, wie sie heute teilweise schon in Bibliotheken vorhanden sind, wo elektronische Dienstleistungen in den physischen Raum integriert sind. In Laboren oder ähnlichen Raumangeboten ist dies sicher einfacher zu gestalten als in klassischen Hörsälen und Seminarräumen. Die Implementierung von Technik in den Raum alleine dürfte hier zu kurz greifen. Es geht um die Interaktionskonzepte an der Schnittstelle von digitaler und physischer Lernwelt. Die Herausforderung für die Hochschulen besteht darin, Experimentierräume zur Verfügung zu stellen, in denen hybride Lehr- und Lernraumstrukturen entwickelt werden können.

\section{Hochschulweite Entwicklung von Raumkonzepten implementieren}

Neben den Quantitäten spielt vor allem die Weiterentwicklung der Qualitäten für Lehr- und Lernräume eine besondere Rolle. Bei aller Virtualität und Digitalisierung ist die Bedeutung des physischen Raums sowohl im Bewusstsein der Hochschulleitungen (Aschinger 2020) wie auch im Erleben der Studierenden sehr groß (Gläser/Kobsch 2020). Eine hochschulweite Konzeptentwicklung zu physischen Lehr- und Lernräumen findet allerdings selten statt (Aschinger 2020). Die strategische Bedeutung von Lehr- und Lernraumkonzepten für den Studienerfolg wird unterschätzt, die Verbindung von Lehre zum selbstbestimmten Lernen nicht offensiv gestaltet. Die Entwicklungen an den Hochschulen sind gegenwärtig vorwiegend durch Einzelinitiativen oder bilaterale Kooperationen (z. B. Bibliothek und Rechenzentrum) geprägt. Hierbei sind Bibliotheken oftmals die „Impulsgeberinnen“, die innovative Konzepte für die Lernräume der Hochschulen einführen (Aschinger 2020). 
Die Gestaltung der physischen Lehr- und Lernräume und die Koordinierung der Nutzung innerhalb einer Hochschule ist - entsprechend der multidimensionalen Struktur - eine komplexe Aufgabe. Die Arbeit an hochschulweiten Konzepten stellt eine Herausforderung dar. Sich damit $\mathrm{zu}$ beschäftigen wird allerdings bei den Hochschulakteurinnen und -akteuren mehr Aufmerksamkeit schaffen, um so mit gebündelten Kräften den möglichen und notwendigen Wandel zu gestalten.

\section{Fazit und Ausblick}

Die Darstellung der Herausforderungen in den Bereichen Hochschulpolitik, Hochschulorganisation, Hochschuldidaktik, digitale Strukturen und physische Lehr- und Lernräume, die sicher nicht alle Aspekte umfasst, macht die komplexe Struktur der Gestaltung der Weiterentwicklung von Hochschulen deutlich. Viele Aspekte sind in den letzten Jahren bereits vielfach in Fachpublikationen thematisiert worden. Der Ansatz des Projektes Lernwelt Hochschule, die Studierendenorientierung in den Fokus $\mathrm{zu}$ stellen, ist dabei bislang nicht sehr intensiv berücksichtigt worden. Die Ergebnisse des Projektes zeigen, dass in der deutschen Hochschullandschaft einige Aktivitäten angestoßen werden, wobei allerdings die ganzheitliche Perspektive auf den Prozess der Weiterentwicklung gelegentlich verloren geht.

Im Projekt wurde allerdings auch deutlich, wie heterogen die deutsche Hochschullandschaft nicht nur vor dem Hintergrund der Ländergesetzgebungen und der Profilbildungen von Hochschulen, sondern auch in Bezug auf die finanzielle Ausstattung, die Größe und nicht zuletzt die Trägerschaft ist. Dieser Vielfalt gilt es bei der Entwicklung von Strategien Rechnung zu tragen. Auch spielt die jeweilige Hochschulkultur eine entscheidende Rolle und Kulturen werden von Menschen geprägt und gelebt. Neben den Studierenden, die nur passagere Stakeholder sind, also als Individuen die Kultur nur punktuell mitprägen können, wenn sie dieses wollen, sind es die internen Stakeholder (Mitarbeitende in allen Bereichen der Hochschule) - und hier sicher besonders die Hochschulleitungen -, die diese Kultur mitprägen und gestalten. Im Forschungsprojekt Lernwelt Hochschule zeigte sich, dass die Innovationsoffenheit bei Hochschulleitungen und auch bei den Lehrenden ein ganz entscheidender Faktor für das Klima einer Hochschule ist.

Auch wenn bürokratische Strukturen in Deutschland sicher an einigen Stellen Innovationsdynamiken bremsen, bleibt doch genügend Spielraum, Innovationen bei der Gestaltung der Lernwelt Hochschule anzugehen und die Grenzen 
zu vermessen. Dass der Bologna-Prozess in Europa sehr unterschiedlich gestaltet wurde, hat sicher auch etwas mit den Bildungskulturen der jeweiligen Länder zu tun. In Deutschland scheint Sicherheit vor Experimentierfreude zu gehen. Innovationen werden oft erst dann eingeführt, wenn sie sich mehrfach bewährt haben. Dass dies ein Widerspruch in sich selbst ist, zeigen vor allem die Länder, die eine andere Innovationskultur haben. Hier wird ausprobiert und wenn es nicht funktioniert, ein neuer Weg beschritten. Scheitern wird eher als Benefit, denn als Nachteil gesehen.

Auch in Deutschland gibt es sicher nicht das Strategiekonzept, das für alle sinnvoll ist. Doch es gibt Korridore für die Entwicklung, die sinnvollerweise zu berücksichtigen sind, wenn die Studierenden ins Zentrum der Überlegungen gestellt werden. Innovationsbereitschaft lässt sich sicher nicht von Seiten der Hochschulpolitik verordnen, doch werden von Seiten der externen Stakeholder, wie zum Beispiel Unternehmen und Organisationen, zunehmend Anforderungen an die Hochschulen herangetragen, nicht nur im Kontext der Forschung neue Wege zu gehen - was Forschung natürlich fast immer gemacht hat -, sondern auch im Kontext der Lehre. Wenn Unternehmen formulieren, dass sie von Hochschulabsolventinnen und -absolventen erwarten, dass diese die Vorgehensweisen und strategische Ausrichtung im Unternehmen hinterfragen und neue Perspektiven einbringen, um auf den dynamisierten Märkten reüssieren zu können, dann sollten dies Studierende an Hochschulen vermittelt bekommen - und diese Anforderungen kommen heute aus allen Bereichen der Gesellschaft.

Doch dies erfordert eine veränderte Hochschulkultur, in deren Rahmen Lehrende ihre Rolle als Mitlernende definieren und die digitalen und physischen Rahmenbedingungen neue Lehr- und Lernformate zulassen. Die Hochschule als Think Tank und Experimentier-Werkstatt für die Zukunft erfordert ein Umdenken, damit es sich bei den Hochschulen in Deutschland nicht ähnlich darstellt wie bei der Automobilindustrie: Über Jahrzehnte wähnte man sich an der Spitze der Entwicklung und sah es als unnötig an, grundlegende Veränderungen vorzunehmen, und plötzlich verändern sich die Strukturen und die Märkte und man muss aufwändig versuchen, verlorenes Terrain wieder zurückzugewinnen.

Wie sich die Zukunft der Lernwelt Hochschule in Deutschland entwickelt, ist schwer vorauszusehen, doch gibt es einige Anzeichen dafür, dass einiges in Bewegung ist. Das Projekt Lernwelt Hochschule konnte hier hoffentlich einige Dimensionen aufzeigen und Herausforderungen herausarbeiten. Den State of the Art darzustellen, war Intention des vorliegenden Bandes. Welche Optionen es für die Zukunft gibt und was auch schon konkret umgesetzt wird, ist dann Thema des Bandes Zukunft Lernwelt Hochschule (Stang/Becker 2020), in dem Hand- 
lungskorridore präsentiert werden, um die derzeitigen Herausforderungen zu meistern.

\section{Literatur}

Aschinger, F. (2020): Konzeption und Management der Lernwelt Hochschule. Herausforderungen und Good Practice aus Sicht der Hochschulakteurinnen und -akteure. In: A. Becker; R. Stang (Hrsg.): Lernwelt Hochschule. Dimensionen eines Bildungsbereichs im Umbruch. Berlin; Boston: De Gruyter Saur, 124-150.

Autorengruppe Bildungsbericht (Hrsg.) (2018): Bildung in Deutschland 2018. Ein indikatorengestützter Bericht mit einer Analyse zu Wirkungen und Erträgen von Bildung. Bielefeld: W. Bertelsmann. https://www.bildungsbericht.de/de/bildungsberichte-seit-2006/bildungsbericht-2018/pdf-bildungsbericht-2018/bildungsbericht-2018.pdf.

Becker, A.; Stang, R. (Hrsg.) (2020a): Lernwelt Hochschule. Dimensionen eines Bildungsbereichs im Umbruch. Berlin; Boston: De Gruyter Saur.

Becker, A.; Stang, R. (2020b): Lernwelt Hochschule im Aufbruch. Zentrale Ergebnisse einer Befragung. In: A. Becker; R. Stang (Hrsg.): Lernwelt Hochschule. Dimensionen eines Bildungsbereichs im Umbruch. Berlin; Boston: De Gruyter Saur, 71-125.

Blättler, A. C; Imhof, F.-D. (2019): Bologna emeritus? 20 Jahre hochschulpolitische Integration Europas. Analyse und Kritik. Bielefeld: wbv.

Dilger, A. (2015): Zurück in die dirigistische Vergangenheit. Das Hochschulzukunftsgesetz in NRW. Diskussionspapier des Instituts für Organisationsökonomik. https://www.econstor. eu/handle/10419/109981.

DINI AG Lernräume (2013): Die Hochschule zum Lernraum entwickeln. Betrachtungen der DINIArbeitsgruppe „Lernräume“. Kassel: Kassel University Press. http://www.uni-kassel.de/ hrz/db4/extern/dbupress/publik/abstract.php?978-3-86219-654-8.

Dohmen, D.; Krempkow, R. (2015): Hochschulautonomie im Ländervergleich: Bestandsaufnahme und Ausblick auf künftige Entwicklungen. Sankt Augustin; Berlin: Konrad-AdenauerStiftung. https://www.kas.de/documents/252038/253252/7_dokument_dok_pdf_42289_1.pdf/3d0a700f-4a00-2e9a-8f30-18eb766d670d? version $=1.0 \& \mathrm{t}=1539652118834$.

Gadatsch, A. (2014): Masterkurs IT-Controlling. Grundlagen und Praxis für IT-Controller und CIOs - Balanced Scorecard - Portfoliomanagement - Wertbeitrag der IT- Projektcontrolling - Kennzahlen - IT-Sourcing - IT-Kosten- und Leistungsrechnung. 5. Aufl. Wiesbaden: Springer Fachmedien.

Gläser, C.; Kobsch, L. (2020): Student Experience in der Lernwelt Hochschule. Studierende im Fokus der Fallstudien. In: A. Becker; R. Stang (Hrsg.): Lernwelt Hochschule. Dimensionen eines Bildungsbereichs im Umbruch. Berlin; Boston: De Gruyter Saur, 151-170.

Günther, D.; Kirschbaum, M; Kruse, R.; Ladwig, T.; Prill, A.; Stang, R.; Wertz, I. (2019): Zukunftsfähige Lernraumgestaltung im digitalen Zeitalter. Thesen und Empfehlungen der Ad-hoc Arbeitsgruppe Lernarchitekturen des Hochschulforum Digitalisierung. Arbeitspapier Nr. 44. Berlin: Hochschulforum Digitalisierung. https://hochschulforumdigitalisierung.de/sites/default/files/dateien/HFD_AP_44-Zukunftsfaehige_Lernraumgestaltung_Web.pdf. 
HHU - Heinrich-Heine-Universität Düsseldorf (2015): Forschungsdaten-Richtlinie der HeinrichHeine-Universität Düsseldorf. https://www.fdm.hhu.de/.

HFD - Hochschulforum Digitalisierung (2016): The Digital Turn - Hochschulbildung im digitalen Zeitalter. Berlin: Edition Stifterverband.

HFD - Hochschulforum Digitalisierung (2019): Digitale Changemaker. Studentische ZukunftsAG zu Hochschulbildung im digitalen Zeitalter. https://hochschulforumdigitalisierung. de/de/themen/digitale-changemaker-studentische-zukunfts-ag-zu-hochschulbildung-imdigitalen-zeitalter.

Hildebrandt, A.; Wolf, F. (Hrsg.) (2016): Die Politik der Bundesländer. Wiesbaden: Springer.

Hüther, O.; Jacob, A. K.; Seidler, H. H.; Wilke, K. (2011): Hochschulautonomie in Gesetz und Praxis. Eine Umsetzungsanalyse vor dem Hintergrund der Förderprogramme des Stifterverbandes und der Heinz Nixdorf Stiftung. Kurzbericht. Essen: Zentrum für Wissenschaftsmanagement.

Koch, S. (2009): Die Bausteine neo-institutionalistischer Organisationstheorie. Begriffe und Konzepte im Lauf der Zeit. In: S. Koch.; M. Schemmann (Hrsg.): Neo-Institutionalismus in der Erziehungswissenschaft. Wiesbaden: VS. 110-132.

Landesregierung NRW (2016a): NRW 4.0. Lernen im digitalen Wandel. https://www.land.nrw/ de/nrw-40-lernen-im-digitalen-wandel.

Landesregierung NRW (2016b): Landeshochschulentwicklungsplan. https://recht.nrw.de/lmi/ owa/br_vbl_show_pdf?p_id=26542.

Niedlich, S. (2020): Neue Ordnung der Bildung. Wiesbaden: Springer VS.

Stang, R. (2017): Analoger Körper im digitalen Raum: Lernen im Zeichen einer ambivalenten Kontextualisierung. In: F. Thissen (Hrsg.): Lernen in virtuellen Räumen: Perspektiven des Mobilen Lernens. Berlin; Boston: De Gruyter Saur, 28-38.

Stang, R.; Becker, A. (Hrsg.) (2020): Zukunft Lernwelt Hochschule. Perspektiven und Optionen für eine Neuausrichtung. Berlin; Boston: De Gruyter Saur.

Weichert, H. (2020a): Dimensionen gesetzlicher Rahmungen. Perspektiven deutscher Hochschulgesetzgebung. In: A. Becker; R. Stang (Hrsg.): Lernwelt Hochschule. Dimensionen eines Bildungsbereichs im Umbruch. Berlin; Boston: De Gruyter Saur, 47-58.

Weichert, H. (2020b): Strukturentwicklungspläne und Leitbilder. Orientierungen für strategische Planungen. In: A. Becker; R. Stang (Hrsg.): Lernwelt Hochschule. Dimensionen eines Bildungsbereichs im Umbruch. Berlin; Boston: De Gruyter Saur, 59-70.

Weichert, H.; Stang, R. (2020): Der Blick von außen. Einschätzungen internationaler Expertinnen und Experten. In: A. Becker; R. Stang (Hrsg.): Lernwelt Hochschule. Dimensionen eines Bildungsbereichs im Umbruch. Berlin; Boston: De Gruyter Saur, 171-182.

Ziegele, F. (2006): Zielvereinbarungen als Kern des „Neuen Steuerungsmodells“. In: Hochschulrektorenkonferenz (Hrsg.): Von der Qualitätssicherung der Lehre zur Qualitätsentwicklung als Prinzip der Hochschulsteuerung. Beiträge zur Hochschulpolitik 1/2006, Band I. Bonn: HRK, 77-105. https://www.hrk.de/fileadmin/redaktion/hrk/02-Dokumente/0210-Publikationsdatenbank/Beitr-2006-01_Von_der_QS_der_Lehre_Bd_I_und_II.pdf.

ZKI - Zentren für Kommunikation und Informationsverarbeitung in Lehre und Forschung (Hrsg.) (2016): Die Prozesslandkarte für den Bereich Studium und Lehre des ZKI AK Campus Management. http://www.zki.de/fileadmin/zki/Publikationen/ZKI_ProzesslandkarteVersion1_-_Mai_2016.pdf. 